\title{
Imaging signatures identified for dementia subtypes
}

Different patterns of brain atrophy
on MRI could identify pathologi-
palty distinct forms of dementia,
patterns of $\quad \begin{aligned} & \text { according to new research. In the } \\ & \text { largest study of its kind, imaging and } \\ & \text { pathology data were used in combi- } \\ & \text { nation to identify imaging signatures } \\ & \text { with different } \\ & \text { pathologies }\end{aligned} \quad \begin{aligned} & \text { that could improve diagnosis of } \\ & \text { dementia. } \\ & \text { Some evidence suggests that } \\ & \text { patterns of brain atrophy are useful } \\ & \text { in the diagnosis of dementia, but } \\ & \text { much of this work has been done } \\ & \text { with patients in whom the pathology } \\ & \text { underlying dementia was unknown. } \\ & \text { The new study, directed by Jonathan } \\ & \text { Schott, included pathology to help } \\ & \text { identify patterns of atrophy that not } \\ & \text { only enable a diagnosis of dementia, } \\ & \text { but also the distinction between } \\ & \text { dementia subtypes. }\end{aligned}$

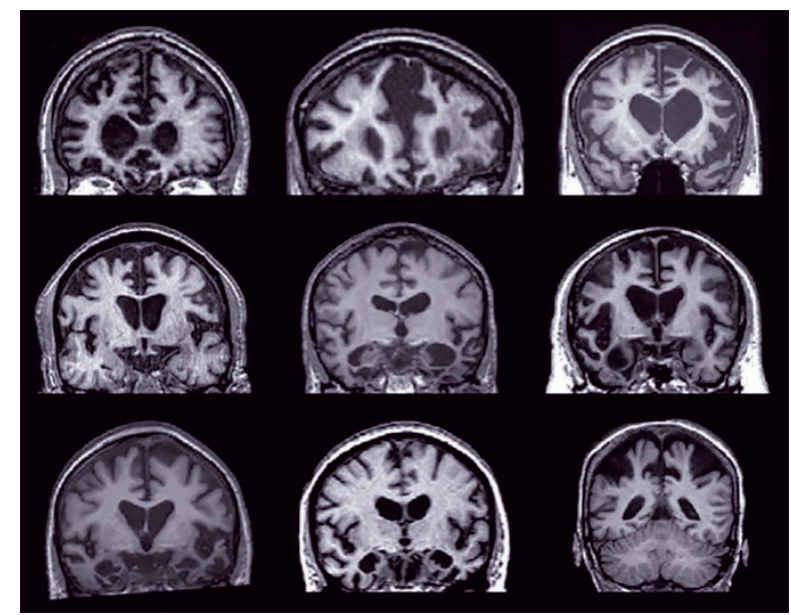

Distinct patterns of atrophy were associated with different underlying pathologies. Image courtesy of L. Harper.
"This work is part of a larger collaboration to pool data from people with a diagnosis of dementia, structural MRI scans acquired during life, and post-mortem confirmation of the underlying pathology," explains Schott. "The aim is to maximize the diagnostic value of structural MRI, which is currently recommended for clinical investigation of dementias."

The study included data from 186 individuals who had pathologically confirmed dementia, and for whom a T1-weighted MRI scan was available. Patients were grouped according to their pathological subtype of dementia: Alzheimer disease (AD; subdivided into early-onset, late-onset and presenilin-1 mutation carriers), dementia with Lewy bodies (DLB), 3-repeat-tau, 4-repeat tau, TDP43 type A and TDP43 type C. In addition, 73 cognitively normal individuals were used as controls.

Voxel-based morphometry (VBM) was used to analyse the MRI scans and compare brain atrophy patterns associated with dementia subtypes. "VBM has been used extensively in the literature, but rarely with such large groups stratified by dementia pathology," says lead author Lorna Harper.

As expected, global brain volume loss was greater among patients with dementia than among cognitively normal controls. However, distinct patterns of atrophy were also associated with different pathologies, with predominant volume loss in different areas: the temperoparietal region in early-onset $\mathrm{AD}$; the focal medial temporal lobe in late-onset $\mathrm{AD}$; the parietal lobe, thalamus, medial temporal lobe and temporal neocortex in presenilin-1 mutant $\mathrm{AD}$; the precentral and postcentral gyri in LBD; and similar but distinct involvement of the frontotemporal lobe in tau and TDP43 pathologies.

"We provide a robust evidence base for structural imaging signals that may aid in the differentiation of dementia subtypes during life," says Harper. "The findings from this study provide much needed validation of the existing literature, which is largely based on clinically stratified groups. However, more work is needed to evaluate the accuracy of these group-level findings at the individual level."

The study did not assess the effects of multiple co-occurring pathologies on patterns of atrophy, but the collaboration is set to continue. "There are plans to add to this dataset to allow us to investigate imaging signatures related to multiple pathologies, and, if funding permits, to make it more widely available to the scientific community," Harper concludes.

Ian Fyfe

ORIGINAL ARTICLE Harper, L. et al. Patterns of atrophy in pathologically confirmed dementias: a voxelwise analysis. J. Neurol. Neurosvurg. Psych. http://dx.doi.org/10.1136/jnnp-2016-314978 (2017) 